\title{
Using Differential Equations to Model Phoretic Parasitism as Part of SCUDEM Challenge
}

\author{
Zachary David Fralish ${ }^{1 *} \mathbb{D}$, Nathan Hallmark ${ }^{1} \mathbb{(}$, Johnathon Marshall ${ }^{1}(\mathbb{C}$
}

${ }^{1}$ Florida Southern College (FSC), USA

*Corresponding Author: zdfralish@gmail.com

Citation: Fralish, Z. D., Hallmark, N., \& Marshall, J. (2021). Using Differential Equations to Model Phoretic Parasitism as Part of SCUDEM Challenge. International Electronic Journal of Mathematics Education, 16(2), em0631. https://doi.org/10.29333/iejme/10889

\begin{tabular}{|c|c|}
\hline ARTICLE INFO & ABSTRACT \\
\hline Received: 25 Mar. 2020 & The SIMIODE Challenge for Undergraduates in Differential Equation Modelling (SCUDEM) offers students the \\
\hline Accepted: 9 Jan. 2021 & $\begin{array}{l}\text { opportunity to improve their mathematical capacity, ability to think critically, and communication skills through } \\
\text { researching, developing, and presenting on a differential equations model for a natural phenomenon. During the } \\
\text { fall } 2019 \text { SCUDEM, we chose to model population dynamics of phoretic parasitism through chemical espionage of } \\
\text { anti-aphrodisiacs by parasitic wasps. Additionally, we modelled the dispersion of anti-aphrodisiac pheromones } \\
\text { from impregnated butterflies and the propagation of resultant action potentials in wasps due to stimulation of } \\
\text { olfactory receptor neurons from these pheromones. This article displays a summary of our undergraduate student } \\
\text { team's response to a provided prompt in SCUDEM IV. This article additionally shares personal testimonies from } \\
\text { three students about the modelling challenge to highlight the benefits of participating in differential equation } \\
\text { modelling challenges. }\end{array}$ \\
\hline
\end{tabular}

Keywords: differential equations, modelling, undergraduate, parasitism

\section{INTRODUCTION}

For the fall 2019 SIMIODE Challenge for Undergraduates in Differential Equation Modelling (SCUDEM), teams of three students were given three phenomena to model from various disciplines that ranged from life sciences to physical sciences to social sciences. Each team was provided with one week to select a problem, build a mathematical model related to the described situation, and prepare both a presentation and an executive summary. The executive summary is a report, confined to two pages, that describes the problem, assumptions, model, implications, and future directions for the model. On the final day of the challenge, all teams met at a local university to present their models and their implications. In the morning, an additional issue to consider was provided and teams were given approximately an hour to modify their model to incorporate this issue for their presentations given that afternoon.

When crafting a successful executive summary, students must digest and critically analyze related literature to gain necessary knowledge about their chosen prompt. Having to develop clear assumptions to simplify real-world phenomena leads students to have a practical outlook when developing a model. The crafting of the model itself engages students in the principles of problembased learning, inductive learning, and inquiry-based learning (Cotic \& Zuljan, 2009). Students also must prepare their presentation, which develops their communication skills. Furthermore, discussions of limitations of their model and future work forces students to ponder wider implications. Ultimately, these students are learning through the creation of their own paradigms, which leads to the retention of more information (Bruning, Shraw, \& Ronning, 1999; Prince \& Fedler, 2006).

Often, simply solving provided equations within the classroom setting does not provide students with enough problem-solving strategies to apply technical skills to real-world contexts that students may be confronted by once they go beyond their college campus. Applying mathematical techniques to scenarios the students have either witnessed or can easily imagine increases the transferability of their newfound knowledge (Rasmussen \& Kwon, 2007). Additionally, it can lead students to have a more positive outlook on the field of mathematics and increase their curiosity about science and technology (Silvia, 2008).

The process of synthesizing ideas into a coherent summary requires students to have a deeper understanding of a particular area of mathematics. Conjointly, presenting their ideas offers students practice communicating to others. Students also work in teams, where each student draws from their distinct backgrounds to advance the group forward. The additional issue that is presented on the day of the challenge leads students to adapt to unforeseen circumstances, which translates directly to the rapidly changing nature of the workplace. Additionally, they can connect with other student teams and build a network that allows for opportunities to further develop their work and relationships. 
The prompt we chose asked students to "develop a mathematical model for the interactions of the male and female $P$. brassicae as well as the parasitic wasps (SCUDEM, 2019)." P. brassicae are a rather social species of large white cabbage butterfly that use chemical signals, known as pheromones, for a variety of interactions (Huigens, Woelke, Pashalidou, Bukovinsky, Smid, \& Fatouros, 2010). After mating, males release an anti-aphrodisiac pheromone onto females to dissuade the pursuit of other males. However, this pheromone, being a kairomone, also attracts the attention of parasitic wasps and allows them to identify butterflies that are likely impregnated. Parasitic wasps are extremely small insects that lay their eggs within the bodies of caterpillars. The implanted wasp larvae use the caterpillar as a food source and eventually consume the caterpillar in its entirety.

This results in two competing pressures on the butterfly population. The anti-aphrodisiacs make it more likely for the males' genes to be passed on and makes females less likely to be bothered by other males allowing them to focus on placing their eggs in the most advantageous place, but also make it more likely that the butterflies' offspring will be eaten by wasp larvae. Provided below in section two is our executive summary that explains our model of the interactions between these species and underlying chemical processes. Our explanation and response to the additional issue is within section three. In section four, we presented three personal testimonies that explain how the challenge influenced and benefited each participant within our team.

\section{EXECUTIVE SUMMARY}

\section{Introduction}

Nature is riddled with contending pressures to pass on genetic information. This evolutionary molding is epitomized by the dynamics between large cabbage white butterfly and parasitic wasps (Forsberg \& Wiklund, 1989; Huigens et al., 2010). After mating, male butterflies secrete anti-aphrodisiac pheromones onto impregnated females (Huigens et al., 2010). Anti-aphrodisiac pheromones are chemicals that prevent further mating. This increases the male's chances of fertilizing a mate and allows the female to focus on caretaking (Forsberg \& Wiklund, 1989). However, phoretic wasps can use these pheromones to their advantage. Phoretic wasps are small parasites that ride on much larger butterflies' backs to lay their eggs within the butterflies' eggs to allow for their eggs feed off of the butterflies' offspring. Through sensing and high-jacking these signals in a form of chemical espionage, the wasps identify impregnated females, hitch a ride to the butterflies' nests, and lay larvae in these nests to parasitize off of the butterfly's eggs (Fatouros et al., 2005; Huigens et al., 2010).

Ultimately, this leads to an interesting trade-off between competing interests. This summary functions to model the long-term population dynamics of butterflies and wasps based on the aforementioned interactions between these species, and to evaluate long-term equilibria based on certain variational parameters. The dispersion of anti-aphrodisiac pheromones from impregnated butterflies and the propagation of resultant action potentials in wasps due to stimulation of olfactory receptor neurons from these pheromones are also modelled.

\section{Assumptions and Models}

In order to succinctly model this natural phenomenon, the following assumptions were made:

- There is no genetic variation in the butterflies that results in changes of pheromone secretion.

- Once a wasp hitches a ride on an impregnated butterfly, if the butterfly survives it will lay a consistent number of eggs and the wasp will infect all eggs resulting in death of all butterfly larvae and the birth of a consistent number of wasps.

- A non-parasitized butterfly will have a consistent number of offspring achieve adulthood.

- There is a consistent percentage of the butterfly population that is impregnated at any time.

- There is no migration in or out of the environment being evaluated; and a wasp only reproduces through the parasitism of a butterfly.

Table 1. Variables and Constants Related to Population Dynamics

\begin{tabular}{|c|c|}
\hline Symbol & Meaning \\
\hline $\mathrm{B}$ & Number of butterflies \\
\hline$B_{\max }$ & Maximum butterfly population \\
\hline W & Number of wasps \\
\hline$d_{B}$ & Death rates of the butterfly population per day \\
\hline $\mathrm{d}_{\mathrm{w}}$ & Death rates of the wasp population per day \\
\hline$\alpha$ & Rate of wasp parasitism \\
\hline$e_{i}$ & Eggs per impregnation \\
\hline We & Number of wasps born per egg parasitized \\
\hline i & Percentage of butterfly population impregnated \\
\hline$I_{w}$ & Number of butterflies that are impregnated and parasitized by a wasp \\
\hline
\end{tabular}

Under these assumptions, the rate of change of the butterfly population can be modelled as

$$
\frac{\partial B}{\partial t}=\left[\left(i B-I_{w}\right) e_{i}-d_{B} B\right]\left(B_{\max }-B\right)
$$

Where $\left(\left(\boldsymbol{i} \boldsymbol{B}-\boldsymbol{I}_{\boldsymbol{w}}\right) \boldsymbol{e}_{\boldsymbol{i}}\right)$ represents the number of butterflies born, $\boldsymbol{d}_{\boldsymbol{B}} \boldsymbol{B}$ represents the number of butterflies that die off, and $\left(\boldsymbol{B}_{\max }-\boldsymbol{B}\right)$ is a logistical correction that caps off the population. The rate of change of the total wasp population and the total number of impregnated butterflies that are under attack from wasps can be modelled as 


$$
\begin{gathered}
\frac{\partial W}{\partial t}=I_{w} e_{i} w_{e}-d_{w} W \\
\frac{\partial I_{w}}{\partial t}=\left(\alpha W-d_{B} I_{w}\right)\left(i B-I_{w}\right)
\end{gathered}
$$

Through this model, the number of impregnated butterflies that are under attack from wasps $\left(\boldsymbol{I}_{\boldsymbol{w}}\right)$ depends on both the butterfly and wasp populations.

Based on observations from performed simulations, three long term phenomena were observed. If the attack rate of the wasps $(\boldsymbol{\alpha})$ is low, the butterfly population will maintain slight oscillations below the carrying capacity while the wasp population stays low. If the attack rate of the wasps is high, all butterflies and then all wasps will eventually die off. If the attack rate is moderate, the populations of the wasps and the butterflies will oscillate back and forth continually.

In addition to the population dynamics, we also model the dynamics of the pheromones spreading from the female butterflies. The anti-aphrodisiac pheromones randomly accelerate (a) outwards from the impregnated butterfly based on the pheromone's mass $(\boldsymbol{m})$ and the drag due to viscosity $(\mathrm{\gamma})$ according to the following equation

$$
a=\frac{\partial v}{\partial t}=-8 / m v
$$

For small spherical objects moving slowly through a viscous fluid, we use the Stokes' drag with drag constant:

$$
\mathrm{\gamma}=6 \pi \eta r
$$

where $\boldsymbol{\eta}$ is the viscosity of air and $\boldsymbol{r}$ is the radius of the pheromones originating from the female butterfly. By integrating (4), we get the velocity and radial position of the pheromones according to time described by the equations:

$$
\begin{gathered}
v=\frac{\partial x}{\partial t}=e^{-m t / \mathrm{\gamma}} \boldsymbol{v}_{0} \\
x=-(\mathrm{\gamma} / m) e^{-m t / \mathrm{\gamma}} v_{0}
\end{gathered}
$$

These released pheromones are registered in the form of electric potentials within olfactory receptor neurons of the antennae of the wasps (Couto, Alenius, \& Dickson, 2005). When the additive value of these electric potentials is greater than some threshold value, an action potential is produced in the neuron that causes an excitatory signal. If this threshold electric potential is not reached, then no signal is formed. The olfactory response system of the wasp consists of olfactory receptor neurons that stimulate synaptic modules that subsequently stimulate motor neurons that then stimulate muscle cells causing a physical response. If we assume that this system has little to no resistance or signal loss, the system can be modelled as a lossless transmission line. The voltage $(\boldsymbol{V})$ and current $(\boldsymbol{I})$ of the propagated electrical signal is given as a function of the self-inductance $(\boldsymbol{L})$ and the capacitance $(\boldsymbol{C})$, respectively, within the neurons. These properties of the electrical signals can be modelled as

$$
\frac{\partial V}{\partial x}=-L \frac{\partial I}{\partial t} \text { and } \frac{\partial I}{\partial x}=-C \frac{\partial V}{\partial t}
$$

Using (8), the standard wave equation

$$
\frac{\partial^{2} V}{\partial t^{2}}-u^{2}\left(\frac{\partial^{2} V}{\partial x^{2}}\right)=\frac{\partial^{2} I}{\partial t^{2}}-u^{2}\left(\frac{\partial^{2} I}{\partial x^{2}}\right)
$$

with propagation speed $\boldsymbol{u}$ of the signal can be solved yielding

$$
u=1 / \sqrt{L C}
$$

\section{Limitations and Future Directions}

Although these equations can describe the aforementioned phenomena, there are several limitations and ways to improve this model. The proposed model for random dispersion of pheromones is only valid for a short time period. Also, the assumptions that all males release the same number of pheromones, all females lay the same number of eggs, and the same number of eggs always achieve adulthood under non-parasitic conditions simplify modelling, but make the model less realistic when describing the variant nature of life. In addition, other factors such as disease, natural disasters, and migration should be incorporated to model population dynamics more realistically.

\section{ADDITIONAL ISSUE}

On the day of the challenge, students were provided with approximately one hour to incorporate an additional phenomenon into their model. The additional issue proposed at SCUDEM IV that we selected was to consider the presence of a common predator to both the parasitic wasps and the white cabbage butterflies in the environment. The praying mantis is one such possible predator that notoriously feeds on butterflies. Assuming the praying mantis population eats both insects at equal rates, the addition of this predator can be modelled by adding an additional term into (1) and (2): 


$$
\begin{gathered}
\frac{\partial B}{\partial t}=\left[\left(i B-I_{w}\right) e_{i}-d_{B} B-d_{P} B\right]\left(B_{\max }-B\right) \\
\frac{\partial W}{\partial t}=I_{w} e_{i} w_{e}-d_{w} W-d_{P} W
\end{gathered}
$$

The new terms, $\boldsymbol{d}_{\boldsymbol{P}} \boldsymbol{B}$ and $\boldsymbol{d}_{\boldsymbol{P}} \boldsymbol{W}$, model the death rates of the butterflies and wasps due to predation of the two species from the praying mantises. The death rate, $\boldsymbol{d}_{\boldsymbol{P}}$, caused by this predator can be modelled as

$$
d_{P}=\alpha_{p} P
$$

where $\boldsymbol{\alpha}_{\boldsymbol{P}}$ is the attack rate of a praying mantis and $\boldsymbol{P}$ is the total population of mantises. From here, the dynamics of the mantis population can be modelled similar to logistic growth as

$$
\frac{\partial P}{\partial t}=P\left(r_{P}-d_{M}\right)\left(P_{\text {max }}-P\right)
$$

where $\boldsymbol{r}_{\boldsymbol{P}}$ is the reproduction rate of the praying mantises, $\boldsymbol{d}_{\boldsymbol{M}}$ is the death rate of praying mantises, and $\boldsymbol{P}_{\boldsymbol{m a x}}$ is the carrying capacity of the praying mantis population. Assuming the population of the praying mantises is only limited by the availability of butterflies and wasps, the maximum population of the praying mantises can be modelled as

$$
P_{\text {max }}=\boldsymbol{k}(B+W)
$$

where $\boldsymbol{k}$ is a proportionality constant.

\section{PERSONAL TESTIMONIES}

"Although I have always enjoyed my mathematics classes, the applications of the course material they discussed has not always been apparent. Participation in a differential equation modelling challenge led me to discover how any natural phenomena could be described in some way by equations. Ultimately, it allowed my perception of math to transition from simply problems in a book into the fundamental governing body of day-to-day life. After I was able to connect material that I learned in my biology and physics classes to the mathematical model that we developed, it became evident just how pertinent mathematics is to any scientific area of study. Participating in such in-depth analysis with a team exposed me to multiple techniques of problem solving and provided me with new methods to approach difficult scenarios. Beyond the math, I made helpful connections with people from various institutions and really enjoyed the challenge. Students with any interest in mathematics should take the opportunity to experience a differential equation modelling challenge."

"Prior to entering the SCUDEM challenges, the highest mathematics course I had taken was Calculus I, and this challenge offered me an opportunity to explore higher level courses, such as Differential Equations, in an extremely unique way. As a college freshman, I had the rewarding experience of not just learning what differential equations are but also learning their application to real-life situations. This is important because college freshmen generally do not have the opportunity to apply their knowledge due to the bulk of their studies being focused on foundational concepts rather than applications of those concepts. In the schooling I have had up to this point, I have studied many subjects superficially, but this challenge gave me the opportunity to see what it was like to study a single and specific topic with extensivity, going narrow and deep versus shallow and wide. One of the really useful skills I learned during the challenge was the process of making limiting assumptions in order to make problems more approachable. Therefore, I fully believe that everyone who can should take part in challenges like this. Whether you are a math major or not, there is still something to be learned and insight to be contributed."

"Participating in the differential equations modelling challenge allowed me to use my creativity to try to effectively model unique, real-world problems. After working with the team to develop the model, I had the opportunity to apply a little bit of computer programming to model our solutions to the problem. Prior to this challenge, I had never personally bridged the gap between mathematical modeling and computer science. In addition to seeing a new way in which mathematics and computer science can overlap, I have made connections with students from other universities that I would not have made otherwise. In fact, I first met one of my closest friends at a SCUDEM challenge last year. From the outlook, I never expected to gain so much from a differential equation modelling challenge. If you are looking for a creative outlet for your mind, if you are looking to make new connections with team members from your school and others, or if you simply want to see what differential equations are all about, I recommend entering a SCUDEM challenge."

Author contributions: All authors have sufficiently contributed to the study, and agreed with the results and conclusions.

Funding: No funding source is reported for this study.

Declaration of interest: No conflict of interest is declared by authors.

Acknowledgements: We would like to thank Jason Elsinger for coaching us prior to the SCUDEM challenge and supporting our academic pursuits within and beyond the classroom. We would also like to thank Brian Winkel and SIMIODE for creating SCUDEM and the Florida Institute of Technology for hosting SCUDEM IV. 


\section{REFERENCES}

Bruning, R. H., Schraw, G. J., \& Ronning, R. R. (1999). Cognitive psychology and instruction. Prentice-Hall, Inc.

Cotič, M., \& Zuljan, M. V. (2009). Problem-based instruction in mathematics and its impact on the cognitive results of the students and on affective-motivational aspects. Educational studies, 35(3), 297-310. https://doi.org/10.1080/03055690802648085

Couto, A., Alenius, M., \& Dickson, B. J. (2005). Molecular, anatomical, and functional organization of the Drosophila olfactory system. Current Biology, 15(17), 1535-1547. https://doi.org/10.1016/j.cub.2005.07.034

Fatouros, N. E., Huigens, M. E., van Loon, J. J., Dicke, M., \& Hilker, M. (2005). Butterfly anti-aphrodisiac lures parasitic wasps. Nature, 433(7027), 704-704. https://doi.org/10.1038/433704a

Forsberg, J., \& Wiklund, C. (1989). Mating in the afternoon: Time-saving in courtship and remating by females of a polyandrous butterfly Pieris napi L. Behavioral Ecology and Sociobiology, 25(5), 349-356. https://doi.org/10.1007/BF00302992

Huigens, M. E., Woelke, J. B., Pashalidou, F. G., Bukovinszky, T., Smid, H. M., \& Fatouros, N. E. (2010). Chemical espionage on species-specific butterfly anti-aphrodisiacs by hitchhiking Trichogramma wasps. Behavioral Ecology, 21(3), 470-478. https://doi.org/10.1093/beheco/arq007

Prince, M. J., \& Felder, R. M. (2006). Inductive teaching and learning methods: Definitions, comparisons, and research bases. Journal of Engineering Education, 95(2), 123-138. https://doi.org/10.1002/j.2168-9830.2006.tb00884.x

Rasmussen, C., \& Kwon, O. N. (2007). An inquiry-oriented approach to undergraduate mathematics. The Journal of Mathematical Behavior, 26(3), 189-194. https://doi.org/10.1016/j.jmathb.2007.10.001

SCUDEM IV 2019 Modeling Problems and Team Submissions. (2019). https://www.simiode.org/groups/scudemivstudents/files

Silvia, P. J. (2008). Interest-The curious emotion. Current Directions in Psychological Science, 17(1), 57-60. https://doi.org/10.1111/j.1467-8721.2008.00548.x 